\title{
Majorana quantization and half- integer thermal quantum Hall effect in a Kitaev spin liquid
}

\section{AUTHOR(S):}

Kasahara, Y.; Ohnishi, T.; Mizukami, Y.; Tanaka, O.; Ma, Sixiao; Sugii, K.; Kurita, N.; ... Motome, Y.; Shibauchi, T.; Matsuda, Y.

\section{CITATION:}

Kasahara, Y. ...[et al]. Majorana quantization and half-integer thermal quantum Hall effect in a Kitaev spin liquid. Nature 2018, 559(7713): 227231

\section{ISSUE DATE:}

2018-07-11

URL:

http://hdl.handle.net/2433/233602

\section{RIGHT:}

This is the accepted manuscript of the article, which has been published in final form at https://doi.org/10.1038/s41586018-0274-0.; The full-text file will be made open to the public on 11 January 2019 in accordance with publisher's 'Terms and Conditions for Self-Archiving'.; This is not the published version. Please cite only the published version.; この論文は 出版社版でありません。引用の際には出版社版をご確認で利用ください。 


\title{
Majorana quantization and half-integer thermal quantum Hall effect in a Kitaev spin liquid
}

\author{
Y. Kasahara ${ }^{1}$, T. Ohnishi ${ }^{1}$, Y. Mizukami ${ }^{2}$, O. Tanaka ${ }^{2}$, Sixiao Ma ${ }^{1}$, K. Sugii ${ }^{3}$, \\ N. Kurita ${ }^{4}$, H. Tanaka ${ }^{4}$, J. Nasu $^{4}$, Y. Motome ${ }^{5}$, T. Shibauchi ${ }^{2}$, and Y. Matsuda ${ }^{1}$ \\ ${ }^{1}$ Department of Physics, Kyoto University, Kyoto 606-8502, Japan \\ ${ }^{2}$ Department of Advanced Materials Science, \\ University of Tokyo, Chiba 277-8561, Japan \\ ${ }^{3}$ Institute for Solid State Physics, University of Tokyo, Chiba 277-8581, Japan \\ ${ }^{4}$ Department of Physics, Tokyo Institute of Technology, \\ Meguro, Tokyo 152-8551, Japan and \\ ${ }^{5}$ Department of Applied Physics, University of Tokyo, Bunkyo, Tokyo 113-8656, Japan
}


The quantum Hall effect (QHE) in two-dimensional (2D) electron gases, which is one of the most striking phenomena in condensed matter physics, involves the topologically protected dissipationless charge current flow along the edges of the sample. Integer or fractional electrical conductance is measured in units of $e^{2} / 2 \pi \hbar$, which is associated with edge currents of electrons or quasiparticles with fractional charges, respectively. Here we discover a novel type of quantization of the Hall effect in an insulating $2 \mathrm{D}$ quantum magnet [1]. In $\alpha$ - $\mathrm{RuCl}_{3}$ with dominant Kitaev interaction on 2D honeycomb lattice [2-7], the application of a parallel magnetic field destroys the long-range magnetic order, leading to a field-induced quantum spin liquid (QSL) ground state with massive entanglement of local spins [8-12]. In the low-temperature regime of the QSL state, we report that the $2 \mathrm{D}$ thermal Hall conductance $\kappa_{x y}^{2 \mathrm{D}}$ reaches a quantum plateau as a function of applied magnetic field. That is, $\kappa_{x y}^{2 \mathrm{D}} / T$ attains a quantization value of $(\pi / 12)\left(k_{B}^{2} / \hbar\right)$, which is exactly half of $\kappa_{x y}^{2 \mathrm{D}} / T$ in the integer QHE. This half-integer thermal Hall conductance observed in a bulk material is a direct signature of topologically protected chiral edge currents of charge neutral Majorana fermions, particles that are their own antiparticles, which possess half degrees of freedom of conventional fermions [13-16]. These signatures demonstrate the fractionalization of spins into itinerant Majorana fermions and $Z_{2}$ fluxes predicted in a Kitaev QSL [1, 3]. Above a critical magnetic field, the quantization disappears and $\kappa_{x y}^{2 \mathrm{D}} / T$ goes to zero rapidly, indicating a topological quantum phase transition between the states with and without chiral Majorana edge modes. Emergent Majorana fermions in a quantum magnet are expected to have a major impact on strongly correlated topological quantum matter.

The topological states of matter are described in terms of topological invariant quantities whose values are quantized. The most popular quantity to prove these states is the electrical Hall conductivity. In the quantum Hall state, the Hall conductance $\sigma_{x y}^{2 \mathrm{D}}$ is quantized in units of $e^{2} / 2 \pi \hbar$ as $\sigma_{x y}^{2 \mathrm{D}}=q\left(e^{2} / 2 \pi \hbar\right)$, where $q$ is integer in integer QHE and is fraction which, with very few exceptions, has an odd denominator in fractional QHE. These quantizations attest to topologically ordered states. Another topological invariant in the topological phase is the $2 \mathrm{D}$ thermal Hall conductance; thermal Hall conductivity per $2 \mathrm{D}$ sheet $\kappa_{x y}^{2 \mathrm{D}}$ is quantized in 
units of $(\pi / 6)\left(k_{B}^{2} / \hbar\right) T$ as

$$
\kappa_{x y}^{2 \mathrm{D}} / T=q(\pi / 6)\left(k_{B}^{2} / \hbar\right)
$$

Although thermal Hall conductivity is much harder to measure than electrical Hall conductivity, it has a clear advantage in revealing the topological phases possessing charge neutral excitations that cannot be detected by the electrical Hall conductivity. In particular, $q=1 / 2$ state with positive thermal Hall sign is a decisive manifestation of the charge neutral edge currents of Majorana particles (Figs. 1a and 1b), distinguishing unambiguously between the different candidate topological orders. We note that a Majorana quantized phase characterized by $q=1 / 2$ has been predicted in chiral topological superconductors [13-15]. However, as the topological superconductivity in bulk materials has not been fully established, previous experiments searching for Majorana fermions have focused on the proximity effect between conventional superconductors and nanowires or topological materials [18-21]. Here we present a fundamentally different approach to this issue and perform direct measurements of the thermal Hall conductance in a bulk insulating magnet.

The systems composed of interacting $1 / 2$ spins on a honeycomb lattice with bonddirectional exchange interactions $J_{K}$ are of vital interest, as they host QSL ground states where topological excitations emerge [1]. This Kitaev QSL exhibits two types of fractionalized quasiparticle excitations, i.e. itinerant (mobile) Majorana fermions and $Z_{2}$ fluxes with a gap. The Majorana fermion has a massless (gapless) Dirac-type dispersion in zero field. In magnetic fields, a novel Majorana fermion system, which is characterized by the bulk gap and gapless edge modes, is realized $[1,3]$, and the $Z_{2}$ flux obeys anyonic statistics.

Recently, a strongly spin-orbit coupled Mott insulator $\alpha-\mathrm{RuCl}_{3}$ has emerged as a prime candidate for hosting an approximate Kitaev QSL. In this compound, local $j_{\text {eff }}=1 / 2$ pseudospins are almost coplanar within the 2D honeycomb layer and the Kitaev interaction $J_{K} / k_{B} \sim 80 \mathrm{~K}$ plays an important role [5-7]. The system is in a spin-liquid (Kitaev paramagnetic) state below $\sim J_{K} / k_{B}$, and shows antiferromagnetic (AFM) order with zigzag spin structure (Fig. 1c) at $T_{N} \approx 7 \mathrm{~K}[22]$ due to non-Kitaev interactions, such as Heisenberg exchange and off-diagonal interactions. The thermal Hall conductance of $\alpha-\mathrm{RuCl}_{3}$ has been measured in magnetic field perpendicular to the 2D planes [23]. For this geometry, finite positive $\kappa_{x y} / T$ emerges in the spin-liquid regime at $T_{N}<T \lesssim 80 \mathrm{~K}$. On entering the AFM state, $\kappa_{x y} / T$ changes the sign and its magnitude is strongly suppressed. The quantization and plateau behaviour of $\kappa_{x y}^{2 \mathrm{D}} / T$ have not been observed in the spin liquid regime. There- 
fore, expanding the measurements to lower temperature region in the liquid state is crucially important.

The response of $\alpha-\mathrm{RuCl}_{3}$ to magnetic fields is highly anisotropic with largely different in-plane and out-of plane properties $[8,11,12,24,25]$. It has been reported that while $T_{N}$ is little influenced by external magnetic field perpendicular to the $2 \mathrm{D}$ plane, $T_{N}$ is dramatically suppressed by the parallel field. This highly anisotropic response is confirmed by the measurements of longitudinal thermal conductivity $\kappa_{x x}$ with the heat current along the $a$ axis in magnetic field $\boldsymbol{H}$ applied along various directions in the $a c$ plane as shown in the inset of Fig. 2a, where $H_{\|}=H \sin \theta$ and $H_{\perp}=H \cos \theta$ are the field component parallel and perpendicular to the $a$ axis, respectively, and $\theta$ is the angle between $\boldsymbol{H}$ and the $c$ axis. In zero field, $\kappa_{x x}$ exhibits a distinct kink at $T_{N}$, as shown in Fig. 2a. While this kink is observed in perpendicular field $\left(\theta=0^{\circ}\right)$ of $12 \mathrm{~T}$ at the same temperature, no kink anomaly is observed in parallel field $\left(\theta=90^{\circ}\right)$ of $7 \mathrm{~T}[11,12]$. In Fig. 2a, we also plot $\kappa_{x x}$ in applied magnetic field of $8 \mathrm{~T}$ tilted away from the $c$ axis $\left(\theta=60^{\circ}, H_{\|} \sim 7 \mathrm{~T}\right)$. Similar to the case of parallel field, no kink anomaly is observed. Figure 1c displays the phase diagram in tilted field of $\theta=60^{\circ}$, where $T_{N}$ is plotted as a function of $H_{\|}$. The inset of Fig. $2 \mathrm{~b}$ shows $T_{N}$ plotted as a function of $H_{\|}$for $\theta=45^{\circ}, 60^{\circ}$ and $90^{\circ}$. While $T_{N}$ for $\theta=60^{\circ}$ well coincides with that for $90^{\circ}$ and vanishes at the same critical field of $H_{\|}^{*} \approx 7 \mathrm{~T}, T_{N}$ for $45^{\circ}$ vanishes at around $H_{\|} \approx 6 \mathrm{~T}$. Although $T_{N}$ does not scale perfectly with $H_{\|}$, these results demonstrate the quasi-2D nature of the magnetic properties. In stark contrast to the strong out-of-plane $(a-c)$ anisotropy, the in-plane $(a-b)$ anisotropy is very small (Extended Data Figs. 3 a, b and c).

Above $H_{\|}^{*}$ where the AFM order melts, the presence of a peculiar spin liquid state has been suggested by the nuclear magnetic resonance (NMR) and neutron scattering measurements. The former reports the presence of spin gap [26] and the latter reveals unusual continuous spin excitations [27]. These magnetic properties are consistent with those expected in a Kitaev-type spin liquid state.

To study the thermal Hall effect in the spin liquid state above $H_{\|}^{*}, \kappa_{x y}$ is measured by sweeping field in tilted directions and obtained by anti-symmetrizing thermal response with respect to the field direction. In this configuration, Hall response is determined by $H_{\perp}$. Since the magnitude of $\kappa_{x y}$ is extremely small compared to $\kappa_{x x}$ in $\alpha-\mathrm{RuCl}_{3}$, special care was taken to detect the intrinsic thermal Hall signal (see Methods). Figures 3a-d and 3e-h 
depict $\kappa_{x y} / T$ at $\theta=60^{\circ}$ and $45^{\circ}$, respectively, plotted as a function of $H_{\perp}$ above $H_{\|}^{*}$ at low temperatures. Experimental error to detect the temperature difference between Hall contacts becomes more significant below $3.5 \mathrm{~K}$, leading to unreliable determination of $\kappa_{x y}$ in our setup.

In the AFM state, $\kappa_{x y} / T$ is extremely small (see Extended Data Fig. 4). Upon entering the field-induced spin liquid state, $\kappa_{x y} / T$, which is positive in sign, increases rapidly. The most striking feature is that $\kappa_{x y} / T$ attains a plateau in the field range of $4.5 \mathrm{~T}<\mu_{0} H_{\perp}<4.8$ $5.0 \mathrm{~T}$ for $\theta=60^{\circ}$ and $6.8 \mathrm{~T}<\mu_{0} H_{\perp}<7.2-7.4 \mathrm{~T}$ for $\theta=45^{\circ}$, as displayed in Figs. 3a-c and 3e-g, respectively. The right axes represent $\kappa_{x y}^{2 \mathrm{D}}$ in units of quantum thermal Hall conductance $(\pi / 6)\left(k_{B}^{2} / \hbar\right) T$, where $\kappa_{x y}^{2 \mathrm{D}}=\kappa_{x y} d$ with the layer distance $d=5.72 \AA[22]$. Remarkably, the plateau is very close to the half of quantum thermal Hall conductance reported in the integer quantum Hall system [17] within the error of $3 \%$, demonstrating the emergence of half-integer thermal Hall conductance plateau. Above $\mu_{0} H_{\perp} \approx 5.0 \mathrm{~T}(7.4 \mathrm{~T})$ for $\theta=60^{\circ}\left(45^{\circ}\right)$, $\kappa_{x y}^{2 \mathrm{D}} / T$ decreases rapidly and vanishes. We note that the half integer quantized plateau is reproduced in crystal from different growth (Extended Data Fig. 5). Although the plateau behaviour seems to be preserved at $5.6 \mathrm{~K}, \kappa_{x y}^{2 \mathrm{D}} / T$ slightly deviates from the quantized value. At higher temperatures, the plateau behaviour disappears (Figs. 3d and 3h).

The temperature dependence of $\kappa_{x y} / T$ at the magnetic fields where the plateau is observed is shown in Fig. 4. The half-integer thermal Hall conductance is observable up to $\sim 5.5 \mathrm{~K}$, above which $\kappa_{x y} / T$ increases rapidly with $T$. As shown in the inset of Fig. $4, \kappa_{x y} / T$ decreases after reaching a maximum at around $15 \mathrm{~K}$ and nearly vanishes above $\sim 60 \mathrm{~K}$ (see Extended Data Fig. 6). As the vanishing temperature of $\kappa_{x y} / T$ is close to Kitaev interaction, it is natural to consider that the finite thermal Hall signal reflects unusual quasiparticle excitations inherent to the spin liquid state governed by the Kitaev interaction (see Methods for more discussion).

In Eq.(1), the coefficient $q$ gives the chiral central charge of the gapless boundary modes, which propagate along one direction. Central charge represents a number of freedom of 1D gapless modes; it is unity for conventional fermions, while it is $1 / 2$ for Majorana fermions whose degrees of freedom is half of conventional fermions. Integer quantum Hall system with the bulk Chern number $\nu$ has $\nu$ boundary modes with $q=\nu$, while a Kitaev QSL with the Chern number $\nu$ has $\nu$ Majorana boundary modes with $q=\nu / 2$. Thus the observed half-integer thermal Hall conductance provides direct evidence of the chiral Majorana edge 
currents. We also note that the positive Hall sign is also consistent with that predicted in the Kitaev QSL [1]. In pure Kitaev model, the excitation energy of $Z_{2}$ flux is estimated to be $\Delta_{F} / k_{B} \sim 0.06 J_{K} / k_{B} \sim 5.5 \mathrm{~K}[7]$. The recent numerical results of the thermal Hall conductance for the 2D pure Kitaev model calculated by the quantum Monte Carlo method show the quantization occurs slightly below $\Delta_{F} / k_{B}[16]$. Experimentally, $\Delta_{F} / k_{B}$ is estimated to be $10 \mathrm{~K}$ [26], which is consistent with the persistence of the thermal Hall quantization up to $\sim 5 \mathrm{~K}$.

In the plateau regime of $\kappa_{x y}$, no anomaly is observed in $\kappa_{x x}$. This is likely because phonon contributions largely dominate over the fermionic excitations arising from spins in $\kappa_{x x}$ in the whole $T$-range $[29,30]$. Moreover, due to the strong spin-phonon coupling in $\alpha-\mathrm{RuCl}_{3}$ [11], the phonon conductivity is expected to show complicated $H$ - and $T$-dependences. The observed plateau behaviours as functions of $H$ and $T$ therefore demonstrate that $\kappa_{x y} / T$ is not affected by the spin-phonon scattering in the plateau regime, providing a strong support for the topological protection. The fact that $\kappa_{x y}$ vanishes at the highest fields as shown in Figs. 3a-c and 3e-g provides direct evidence that the thermal Hall effect is not influenced by phonons, demonstrating that $\kappa_{x y}$ is a unique and powerful probe in the search for Majorana quantization.

We stress that the half-integer thermal Hall conductance in a bulk material is a direct consequence of the chiral Majorana edge current. Recent experiments based on the proximity effect between a quantum anomalous Hall insulator and a conventional superconductor have reported a signature of chiral Majorana edge modes [21]. However, this is based on the observation of half-integer quantization of the longitudinal electrical conductance via the scattering matrix effect between the edge states of the insulator and superconductor. Moreover, Majorana fermions in the Kitaev magnets and topological superconductors have essentially different aspects. In the former, strong correlations give rise to the emergent Majorana fermions, while in the latter they do not play a role. In addition, Majorana fermions exist inside the bulk of a sample in the Kitaev QSL state, in sharp contrast to topological superconductors where they appear only at the edges. The distinct nature is presumably supported by the fact that the quantum plateau disappears below $\sim 400 \mathrm{mK}$ in the topological superconductor device [21], whereas it is preserved up to $\sim 5 \mathrm{~K}$ in $\alpha-\mathrm{RuCl}_{3}$.

At $\theta=60^{\circ}, \kappa_{x y}^{2 \mathrm{D}}(H) / T$ increases slightly from the quantized value before going to zero at high field at 4.3 and $4.9 \mathrm{~K}$, which is reproduced in a different crystal (Extended Data 
Fig. 5a). However, such a behaviour is not observed for $\theta=45^{\circ}$. On the other hand, the overshoot behaviour is also observed in the temperature dependence irrespective of the angle (Fig. 4) and crystal (Extended Data Fig. 5b), and thus, there seem to be certain highenergy corrections that are responsible for the excess conductivity at high fields and high temperatures. These overshoot behaviours are in contrast to the numerical results of the thermal Hall effect for the 2D pure Kitaev model with a weak magnetic field [16]. Meanwhile, it has been pointed out that non-Kitaev interactions, such as Heisenberg and off-diagonal ones, are important for $\alpha-\mathrm{RuCl}_{3}[31,37]$. Hence, the discrepancy may be attributed to high-field effects and/or non-Kitaev interactions, which deserves further study.

The nearly vanishing of $\kappa_{x y}^{2 \mathrm{D}} / T$ after the rapid suppression in the high-field regime (Figs. 3a-c and 3e-g) demonstrates the disappearance of chiral Majorana edge currents. As shown by the open blue square in Fig. 1b, the temperature at which $\kappa_{x y}^{2 \mathrm{D}} / T$ vanishes decreases rapidly with decreasing $\mu_{0} H_{\|}$. This suggests a topological quantum phase transition from the non-trivial QSL to trivial high-field state, where the thermal Hall effect is absent, at $\mu_{0} H_{\|} \sim 9 \mathrm{~T}$ as shown by the red circle in Fig. 1c [32]. Specific heat at $0.47 \mathrm{~K}$ for $\theta=60^{\circ}$ exhibits a dip-like anomaly in the vicinity of $9 \mathrm{~T}$, which can be associated with an abrupt change of the spin gap at the topological transition, providing strong support on the presence of characteristic field revealed by $\kappa_{x y} / T$ (Extended Data Figs. 7a, b and c). The vanishing of $\kappa_{x y} / T$ at the highest fields is unlikely due to the crossover to a simple forced ferromagnetic state because the magnetization at $9 \mathrm{~T}$ is less than $1 / 3$ of the fully polarized value, indicating there still remain paramagnetic spins. The observation of halfinteger thermal Hall conductance reveals that topologically protected chiral Majorana edge currents persist in $\alpha-\mathrm{RuCl}_{3}$, even in the presence of non-Kitaev interactions and parallel field. The observation opens a possibility to link to non-Abelian anyons important for the topological quantum computing, revealing novel aspects of strongly correlated topological quantum matters.

[1] Kitaev, A. Anyons in a exactly solved model and beyond. Ann. Phys. 321, 2-111 (2006).

[2] Jackeli, G. \& Khaliullin, G. Mott Insulators in the Strong Spin-Orbit Coupling Limit: From Heisenberg to a Quantum Compass and Kitaev Models. Phys. Rev. Lett. 102, 017205 (2009). 
[3] Trebst, S. Kitaev Materials. Preprint at http://arXiv.org/cond-mat/1701.07056 (2017).

[4] Kim, H.-S., Shankar, V. V., Catuneanu, A. \& Kee, H.-Y. Kitaev magnetism in honeycomb $\mathrm{RuCl}_{3}$ with intermediate spin-orbit coupling. Phys. Rev. B 91, 241110(R) (2015).

[5] Banerjee, A. et al. Proximate Kitaev quantum spin liquid behaviour in a honeycomb magnet. Nat. Mat. 15, 733-740 (2016).

[6] Sandilands, L. J., Tian, Y., Plumb, W., Kim, Y.-J. \& Burch, K. S. Scattering Continuum and Possible Fractionalized Excitations in $\alpha-\mathrm{RuCl}_{3}$. Phys. Rev. Lett. 114, 147201 (2015).

[7] Nasu, J., Knolle, J., Kovrizhin, D. L., Motome, Y. \& Moessner, R. Fermionic response from fractionalization in an insulating two-dimensional magnet. Nat. Phys. 12, 912-915 (2016).

[8] Yadav, R., Bogdanov, N. A., Katukuri, V. M., Nishimoto, S., van der Brink, J., \& Hozoi, L., Kitaev exchange and field-induced quantum spin-liquid states in honeycomb $\alpha-\mathrm{RuCl}_{3} . S c i$. Rep. 6, 37925 (2016).

[9] Baek, S.-H. et al. Evidence for a Field-Induced Quantum Spin Liquid in $\alpha-\mathrm{RuCl}_{3}$. Phys. Rev. Lett. 119, 037201 (2017).

[10] Wolter, A. U. B. et al. Field-induced quantum criticality in the Kitaev system $\alpha-\mathrm{RuCl}_{3}$. Phys. Rev. B 96, 041405(R) (2017).

[11] Leahy, I. A., Pocs, C. A., Siegfried, P. E., Graf, D., Do, S.-H., Choi, K.-Y., Normand, B. \& Lee, Minhyea. Anomalous Thermal Conductivity and Magnetic Torque Response in the Honeycomb Magnet $\alpha-\mathrm{RuCl}_{3}$. Phys. Rev. Lett. 118, 187203 (2017).

[12] Hentrich, R. et al. Large field-induced gap of Kitaev-Heisenberg paramagnons in $\alpha-\mathrm{RuCl}_{3}$, Preprint at http://arXiv.org/cond-mat/1703.08623 (2017).

[13] Read, N. \& Green, D. Paired states of fermions in two dimensions with breaking of parity and time-reversal symmetries and the fractional quantum Hall effect. Phys. Rev. B 61, 10267-10297 (2000).

[14] Sumiyoshi, H. \& Fujimoto, S. Quantum Thermal Hall Effect in a Time-Reversal-SymmetryBroken Topological Superconductor in Two Dimensions: Approach from Bulk Calculations. J. Phys. Soc. Jpn. 82, 023602 (2013).

[15] Nomura, K., Ryu, S., Furusaki, A. \& Nagaosa, N. Cross-Correlated Responses of Topological Superconductors and Superfluids, Phys. Rev. Lett. 108, 026802 (2012).

[16] Nasu, J., Yoshitake, J. \& Motome, Y. Thermal Transport in the Kitaev Model. Phys. Rev. Lett. 119, 127204 (2017). 
[17] Banerjee, M., Heiblum, M., Rosenblatt, A., Oreg, Y., Feldman, D. E., Stern, A. \& Umansky, V. Observed quantization of anyonic heat flow. Nature 545, 75-79 (2017).

[18] Mourik, V., Zuo, K., Frolov, S. M., Plissard, S. R., Bakkers, E. P. A. M. \& Kouwenhoven, L. P. Signatures of Majorana Fermions in Hybrid superconductor-Semiconductor Nanowire Devices. Science 336, 1003-1007 (2012).

[19] Nadj-Perge, S., Drozdov, I. K., Li, J., Chen, H., Jeon, S., Seo, J., MacDonald, A. H., Bernevig, B. A. \& Yazdani, A. Observation of Majorana fermions in ferromagnetic atomic chains on a superconductor. Science 346, 602-607 (2014).

[20] Das, A., Ronen, Y., Most, Y., Oreg, Y., Heiblum, M. \& Shtrikman, H. Zero-bias peaks and splitting in an Al-InAs nanowire topological superconductor as a signature of Majorana fermions. Nat. Phys. 8, 887-895 (2012).

[21] He, Q. L. et al. Chiral Majorana fermion modes in a quantum anomalous Hall insulatorsuperconductor structure. Science 357, 294-299 (2017).

[22] Johnson, R. D. et al. Monoclinic crystal structure of $\alpha-\mathrm{RuCl}_{3}$ and the zigzag antiferromagnetic ground state. Phys. Rev. B 92, 234119 (2015).

[23] Kasahara, Y. et al. Thermal Hall effect in a Kitaev spin liquid: A possible signature of Majorana chiral edge current. Preprint at http://arXiv.org/cond-mat/1709.10286 (2017).

[24] Majumder, M., Schmidt, M., Rosner, H., Tsirlin, A. A., Yasuoka, H. \& Baenitz, M. Anisotropic $\mathrm{Ru}^{3+} 4 d^{5}$ magnetism in the $\alpha-\mathrm{RuCl}_{3}$ honeycomb system: Susceptibility, specific heat, and zerofield NMR. Phys. Rev. B 91, 180401(R) (2015).

[25] Chaloupka, L. \& Khaliullin, G. Magnetic anisotropy in the Kitaev model systems $\mathrm{Na}_{2} \mathrm{IrO}_{3}$ and $\mathrm{RuCl}_{3}$. Phys. Rev. B 94, 064435 (2016).

[26] Janša, N. et al. Observation of gapped anyons in the Kitaev honeycomb magnet under a magnetic field. Preprint at http://arXiv.org/cond-mat/1706.08455 (2017).

[27] Banergee, A. et al. Excitations in the field-induced quantum spin liquid state of $\alpha-\mathrm{RuCl}_{3}$. Preprint at http://arXiv.org/cond-mat/1706.07003 (2017).

[28] Zheng, J. et al. Gapless Spin Excitations in the Field-Induced Quantum Spin Liquid Phase of $\alpha-\mathrm{RuCl}_{3}$. Phys. Rev. Lett. 119, 227208 (2017).

[29] Hirobe, D., Sato, M. Shiomi, Y, Tanaka, H, \& Saitoh, E. Magnetic thermal conductivity far above the Néel temperatures in the Kitaev-magnet candidate $\alpha-\mathrm{RuCl}_{3}$. Phys. Rev. B 95, $241112(2017)$. 
[30] Yu, Y. J., Xu, Y., Ran, K. J., Ni, J. M., Huang, Y. Y., Wen, J. S. \& Li, S. Y. Ultralowtemperature thermal conductivity of the Kitaev honeycomb magnet $\alpha-\mathrm{RuCl}_{3}$ across the fieldinduced phase transition. Preprint at http://arXiv.org/cond-mat/1708.04090 (2017).

[31] Gohlke, M., Wachtel, G., Yamaji, Y., Pollmann, F., \& Kim, Y. B. Signatures of Quantum Spin Liquid in Kitaev-like Frustrated Magnets. Phys. Rev. B 97, 075126 (2018).

[32] Jiang, H-C., Gu, Z-C., Qi, X-L., \& Trebst, S. Possible proximity of the Mott insulating iridate $\mathrm{Na}_{2} \mathrm{IrO}_{3}$ to a topological phase: Phase diagram of the Heisenberg-Kitaev model in a magnetic field. Phys. Rev. B 83, 245104 (2011).

\section{ACKNOWLEDGEMENTS}

We thank S. Fujimoto, H. Ishizuka, N. Kawakami, H.-Y. Kee, Y. B. Kim, E.-G. Moon, N. P. Ong, M. Shimozawa, M. Udagawa, and M. Yamashita for useful discussions. This work was supported by Grants-in-Aid for Scientific Research (KAKENHI) (No. 25220710, 15H02014, 15H02106, 15H05457, and 15K17692) and Grants-in-Aid for Scientific Research on innovative areas "Topological Materials Science" (No. JP15H05852) from Japan Society for the Promotion of Science (JSPS), and by Yamada Science Foundation and Toray Science Foundation.

\section{AUTHOR CONTRIBUTIONS}

Y.K. and Y.Matsuda conceived and designed the study. Y.K., T.O. and S.M. performed the thermal transport measurements. Y.Mizukami, O.T., and K.S. performed the specific heat measurements. N.K. and H.T. synthesized the high-quality single crystalline samples. Y.K., T.O., J.N., Y.Motome, T.S., and Y.Matsuda discussed the results. Y.K., J.N., Y.Motome, T.S., and Y.Matsuda prepared the manuscript.

\section{AUTHOR INFORMATION}

Reprints and permissions information is available at www.nature.com/reprints. The authors declare no competing financial interests. Readers are welcome to comment on the online version of the paper. Correspondence and requests for materials should be addressed 
to Y.Matsuda (matsuda@scphys.kyoto-u.ac.jp).

\section{MAIN FIGURE LEGENDS}

Figure 1 | Chiral Majorana edge currents and temperature-magnetic field phase

diagram of $\alpha-\mathbf{R u C l}_{3}$. a,b, Schematic illustrations of heat conductions in the integer quantum Hall state of 2D electron gas (a) and Kitaev QSL state (b) in magnetic field applied perpendicular to the planes (gray arrows). In the red (blue) regime, the temperature is higher (lower). The red and blue arrows represent thermal flow. In the quantum Hall state, the skipping orbits of electrons (green spheres) at the edge, which form 1D edge channels, conduct heat and $\kappa_{x y}$ is negative in sign. In the Kitaev QSL state, spins are fractionalized into Majorana fermions (yellow spheres) and $Z_{2}$ fluxes (black hexagons). The heat is carried by chiral edge currents of charge neutral Majorana fermions and $\kappa_{x y}$ is positive in sign. c, Phase diagram of $\alpha-\mathrm{RuCl}_{3}$ in tilted field of $\theta=60^{\circ}$. Open and closed diamonds represent the onset temperature of AFM order with zigzag type $T_{N}$ determined by $T$ - and $H$-dependences of $\kappa_{x x}$, respectively (see Fig. 2b and Extended Data Figs. 1 and 2). Below $T \sim J_{K} / k_{B}$, the spin liquid (Kitaev paramagnetic) state appears. At $\mu_{0} H_{\|}^{*} \sim 7 \mathrm{~T}, T_{N}$ vanishes (blue arrow). A half-integer quantized plateau of 2D thermal Hall conductance is observed in the red regime. Open blue squares represent the fields at which the thermal Hall response disappears. Red circle is the suggested topological phase transition point that separates the non-trivial QSL state with topologically protected chiral Majorana edge currents and a trivial state, such as non-topological spin liquid. The striped region denotes the region that was not accessible in the thermal Hall effect measurements. Error bars represent one standard deviation.

Figure $2 \mid$ Longitudinal thermal conductivity in $\alpha-\mathbf{R u C l}_{3}$. a, Temperature dependence of $\kappa_{x x}$ in magnetic field $\boldsymbol{H}$ applied along various directions in the ac plane. Inset illustrates a schematic of the measurement setup for $\kappa_{x x}$ and $\kappa_{x y}$. b, $\kappa_{x x}$ at $\theta=60^{\circ}$ plotted as a function of parallel field component $H_{\|}$. Inset shows $T_{N}$ vs. $H_{\|}$at different field directions. $T_{N}$ is determined by the $T$-dependence of $\kappa_{x x}$ shown in Fig. 2a (open symbols) and by the minimum in the $H$-dependence of $\kappa_{x x}$ (filled symbols) shown by arrows in the main panel. The crosses are $T_{N}$ for $\theta=90^{\circ}$ determined from magnetic susceptibility measurements [27]. Figure 3 | Half-integer thermal Hall conductance plateau. a-h, Thermal Hall con- 
ductivity $\kappa_{x y} / T$ in tilted field of (a-d) $\theta=60^{\circ}$ and (e-h) $45^{\circ}$ (see inset of Fig. 2a) plotted as a function of $H_{\perp}$. The top axes show the parallel field component $H_{\|}$. The right scales represent the $2 \mathrm{D}$ thermal Hall conductance $\kappa_{x y}^{2 \mathrm{D}} / T$ in units of $(\pi / 6)\left(k_{B}^{2} / \hbar\right)$. Violet dashed lines represent the half-integer thermal Hall conductance, $\kappa_{x y}^{2 \mathrm{D}} /\left[T(\pi / 6)\left(k_{B}^{2} / \hbar\right)\right]=1 / 2$. Error bars represent one standard deviation.

Figure 4 Temperature dependence of the thermal Hall conductance. The main panel shows $\kappa_{x y} / T$ in tilted fields of $\theta=45^{\circ}$ and $60^{\circ}$ (see inset of Fig. $2 \mathrm{a}$ ) at $\mu_{0} H_{\perp}=7 \mathrm{~T}$ and $4.6 \mathrm{~T}$, respectively, at which quantized thermal Hall conductance plateau is observed at low temperatures. The right scale is the $2 \mathrm{D}$ thermal Hall conductance $\kappa_{x y}^{2 \mathrm{D}} / T$ in units of $(\pi / 6)\left(k_{B}^{2} / \hbar\right)$. Violet dashed line represents the half-integer thermal Hall conductance, $\kappa_{x y}^{2 \mathrm{D}} /\left[T(\pi / 6)\left(k_{B}^{2} / \hbar\right)\right]=1 / 2$. Inset shows the same data in a wider temperature regime. Error bars represent one standard deviation.

\section{METHODS}

Single crystal growth. High-quality single crystals of $\alpha-\mathrm{RuCl}_{3}$ were grown by a vertical Bridgman method as described in Ref. [33]. For thermal transport measurements, we carefully picked up thin crystals with plate-like shape. Typical sample size is $\sim$ $2 \mathrm{~mm} \times 0.5 \mathrm{~mm} \times 0.02 \mathrm{~mm}$. We selected the best crystal in which no anomaly associated with the magnetic transition at $14 \mathrm{~K}$ due to the stacking faults is detected by magnetic susceptibility, specific heat, and thermal transport measurements.

Thermal transport measurements. Thermal and thermal Hall conductivities were measured simultaneously on the same crystal by the standard steady state method, using the experimental setup illustrated in the inset of Fig. 2a. Heat current $\boldsymbol{q}$ were applied along the $a$ axis $(\boldsymbol{q} \| \boldsymbol{x})$. Using special jigs, magnetic field $\boldsymbol{H}$ is applied along various directions in the ac plane within an accuracy less than one degree (inset of Fig. 2a). The temperature gradient $-\nabla_{x} T \| \boldsymbol{x}$ and $-\nabla_{y} T \| \boldsymbol{y}$ was measured by carefully calibrated Cernox thermometers. Sample temperature was measured with accuracy within $0.1 \mathrm{mK}$ using alternating current resistance bridges. $1 \mathrm{k} \Omega$ chip resistor was used to generate the heat current. The magnitude of thermal gradient is less than $5 \%$ of the base temperature. To reduce the noise level, all measurements are performed in the radio-frequency shielded room. For the measurements of the thermal Hall effect, we removed the longitudinal response due to misalignment of the contacts 
by anti-symmetrizing the measured $\nabla_{y} T$ as $\nabla_{y} T^{\operatorname{asym}}(H)=\left[\nabla_{y} T(H)-\nabla_{y} T(-H)\right] / 2$ at each temperature. We note that the offset transverse thermal gradient due to the misalignment of Hall contact was reduced to be less than $0.5 \%$ of longitudinal thermal gradient in zero field. $\kappa_{x x}$ and $\kappa_{x y}$ were obtained from longitudinal thermal resistivity $w_{x x}=\nabla_{x} T / q$ and thermal Hall resistivity $w_{x y}=\nabla_{y} T^{\mathrm{asym}} / q$ as $\kappa_{x x}=w_{x x} /\left(w_{x x}^{2}+w_{x y}^{2}\right)$ and $\kappa_{x y}=w_{x y} /\left(w_{x x}^{2}+w_{x y}^{2}\right)$. To avoid background Hall signal, a LiF heat bath and non-metallic grease were used. We confirmed that the thermal Hall signal in LiF is negligibly small within our experimental resolution [35]. The experimental error in determining $\kappa_{x y}$ caused by the uncertainty in measuring the distance between the contacts and the thickness of the crystal is within $\pm 2 \%$.

Specific heat measurements. Specific heat was measured by a long relaxation method [34] in a ${ }^{3}$ He cryostat. A Cernox chip resistor was used as both thermometer and heater. The sample was attached to the calorimeter with grease. The thermometer was calibrated in magnetic field up to $12 \mathrm{~T}$.

Origin of thermal Hall response. We here discuss $\kappa_{x y} / T$ in the high temperature spin liquid regime where no plateau behaviour is observed. Finite $\kappa_{x y} / T$ in the spin liquid states has been reported only in kagomé insulator volborthite $\mathrm{Cu}_{3} \mathrm{~V}_{2} \mathrm{O}_{7}(\mathrm{OH})_{2} \cdot 2 \mathrm{H}_{2} \mathrm{O}$ so far [35]. We point out that the behaviour of $\kappa_{x y} / T$ in the high temperature regime of $\alpha-\mathrm{RuCl}_{3}$ is essentially different from that in the liquid state of volborthite; $\kappa_{x y}$ of volborthite is opposite in sign to $\alpha-\mathrm{RuCl}_{3}$ and its magnitude is more than one order magnitude smaller. All theories until now, except for Kitaev model, predict that the finite $\kappa_{x y}$ in spin liquid states can appear when the Dzyaloshinsky-Moriya (DM) interaction is present [36]. In fact, volborthite has a large $\mathrm{DM}$ interaction. However, the $\mathrm{DM}$ interaction in $\alpha-\mathrm{RuCl}_{3}$ is approximately $5 \mathrm{~K}$, which is much smaller than $J_{K}$ [37], and hence it does not play an important role at high temperatures. Moreover, the phonon thermal Hall conductivity is three orders of magnitude smaller than the observed $\kappa_{x y} / T$ in the spin-liquid state and shows essentially different T-dependence [38].

[33] Kubota, Y., Tanaka, H., Ono, T., Narumi, Y. \& Kindo, K. Successive magnetic phase transition in $\alpha-\mathrm{RuCl}_{3}$ : XY-like frustrated magnet on the honeycomb lattice. Phys. Rev. B 91, 094422 (2015). 
[34] Taylor, O.J., Carrington, A. \& Schlueter, J. A. Specific-Heat Measurements of the Gap Structure of the Organic Superconductor $\kappa-(\mathrm{ET})_{2} \mathrm{Cu}\left[\mathrm{N}(\mathrm{CN})_{2}\right] \mathrm{Br}$ and $\kappa-(\mathrm{ET})_{2} \mathrm{Cu}(\mathrm{NCS})_{2}$. Phys. Rev. Lett. 99, 057001 (2007).

[35] Watanabe, D. et al. Emergence of nontrivial magnetic excitations in a spin liquid state of kagomé volborthite. Proc. Natl. Acad. Sci. USA 113, 8653-8657 (2016).

[36] Han, J. H. \& Lee, H. Spin Chirality and Hall-Like Transport Phenomena of Spin Excitations. J. Phys. Soc. Jpn. 86, 011007 (2016).

[37] Winter, S. M., Li, Y., Jeschke, H.O. \& Valentí, R. Challenges in design of Kitaev materials: Magnetic interactions from competing energy scales. Phys. Rev. B 93, 214431 (2016).

[38] Sugii, K. et al. Thermal Hall effect in a phonon-glass $\mathrm{Ba}_{3} \mathrm{CuSb}_{2} \mathrm{O}_{9}$. Phys. Rev. Lett. 118, 145902 (2017).

Data availability. The data that support the plots within this paper and other findings of this study are available from the corresponding author upon reasonable request.

\section{EXTENDED DATA FIGURE LEGENDS}

\section{Extended Data Figure 1 Temperature dependence of the longitudinal thermal} conductivity. a,b, $\kappa_{x x}$ in tilted field of (a) $\theta=60^{\circ}$ and (b) $45^{\circ}$ (see inset of Fig. 2a) plotted as a function of temperature. Arrows indicate the onset temperature of the AFM order $T_{N}$. Extended Data Figure 2 | Field dependence of the longitudinal thermal conductivity. a,b, $\kappa_{x x}$ in tilted field of (a) $\theta=60^{\circ}$ and (b) $45^{\circ}$ (see inset of Fig. 2a) plotted as a function of parallel field component $H_{\|}$. Arrows indicate the minimum of $\kappa_{x x}$, which is attributed to the onset field of the AFM order.

Extended Data Figure $3 \mid$ Phase diagram of $\alpha-\mathbf{R u C l}_{3}$ for $\boldsymbol{H} \| a$ and $\boldsymbol{H} \| b$. a,b, Temperature dependence of the specific heat divided by $T, C / T$ for (a) $\boldsymbol{H} \| a$ and (b) $\boldsymbol{H} \| b$. Arrows indicate the Neel temperature $T_{N}$. c, Field dependence of $T_{N}$ for $\boldsymbol{H} \| a$ and $\boldsymbol{H} \| b$ determined by the specific heat measurements. $T_{N}$ determined by the thermal conductivity and magnetic susceptibility [27] is also shown. The critical field for $\boldsymbol{H} \| a$ is slightly lower than that for $\boldsymbol{H} \| b$, but both phase diagrams are very similar.

Extended Data Figure 4 | Field dependence of thermal Hall conductivity. a,b, Thermal Hall conductivity $\kappa_{x y} / T$ in tilted field of (a) $\theta=60^{\circ}$ and (b) $45^{\circ}$ (see inset of 
Fig. 2a) plotted as a function of $H_{\perp}$. The top axes show the parallel field component $H_{\|}$. The right scales represent the $2 \mathrm{D}$ thermal Hall conductance $\kappa_{x y}^{2 \mathrm{D}} / T$ in units of $(\pi / 6)\left(k_{B}^{2} / \hbar\right)$. Violet dashed lines represent the half-integer thermal Hall conductance, $\kappa_{x y}^{2 \mathrm{D}} /\left[T(\pi / 6)\left(k_{B}^{2} / \hbar\right)\right]=1 / 2$. Error bars represent one standard deviation.

Extended Data Figure 5 | Sample dependence of $\boldsymbol{\kappa}_{x y}$. a, $\kappa_{x y} / T$ measured in a different crystal (sample $\# 2$ ) for $\theta=60^{\circ}$ (see inset of Fig. 2a) at $4.3 \mathrm{~K}$ plotted as a function of $H_{\perp}$. The right scales represent the $2 \mathrm{D}$ thermal Hall conductance $\kappa_{x y}^{2 \mathrm{D}} / T$ in units of $(\pi / 6)\left(k_{B}^{2} / \hbar\right)$. The half-integer thermal Hall conductance plateau is observed at $4.5 \mathrm{~T}<\mu_{0} H_{\perp}<5.0 \mathrm{~T}$. The field where the overshoot behavior from the quantization value is observed is slightly higher than that of sample $\# 1$, but the field at which $\kappa_{x y} / T$ vanishes $\left(\mu_{0} H_{\|} \sim 9.3 \mathrm{~T}\right)$ is close to that of sample $\# 1$. $\mathbf{b}, \kappa_{x y} / T$ of sample $\# 2$ in tilted field of $\theta=60^{\circ}$ plotted as a function of $H_{\perp}$ at $11 \mathrm{~K}$. Error bars represent one standard deviation.

Extended Data Figure 6 | Field dependence of thermal Hall conductivity in tilted fields at high temperatures. a-d, Thermal Hall conductivity $\kappa_{x y} / T$ in tilted field of $(\mathbf{a}, \mathbf{b})$ $\theta=60^{\circ}$ and $(\mathbf{c}, \mathbf{d}) 45^{\circ}$ (see inset of Fig. 2a) plotted as a function of $H_{\perp}$. The right scales represent the $2 \mathrm{D}$ thermal Hall conductance $\kappa_{x y}^{2 \mathrm{D}} / T$ in units of $(\pi / 6)\left(k_{B}^{2} / \hbar\right)$. Violet dashed lines represent the half-integer thermal Hall conductance, $\kappa_{x y}^{2 \mathrm{D}} /\left[T(\pi / 6)\left(k_{B}^{2} / \hbar\right)\right]=1 / 2$. Error bars represent one standard deviation.

Extended Data Figure 7 | Specific heat above $\boldsymbol{H}_{\|}^{*}$ a,b, Temperature dependence of $C / T$ for $(\mathbf{a}) \theta=60^{\circ}(\boldsymbol{H}$ is tilted within the $a c$ plane $)$ and (b) $90^{\circ} . \mathbf{c}, C / T$ at $0.47 \mathrm{~K}$ plotted as a function of $H_{\|}$for $\theta=60^{\circ}$ and $90^{\circ}$. $C(H) / T$ exhibits a dip-like anomaly for $\theta=60^{\circ}$ and a kink anomaly for $\theta=90^{\circ}$ at $\mu_{0} H_{\|} \sim 9.2 \mathrm{~T}$ (dashed line). This field almost coincides with the characteristic field at which $\kappa_{x y} / T$ vanishes. 

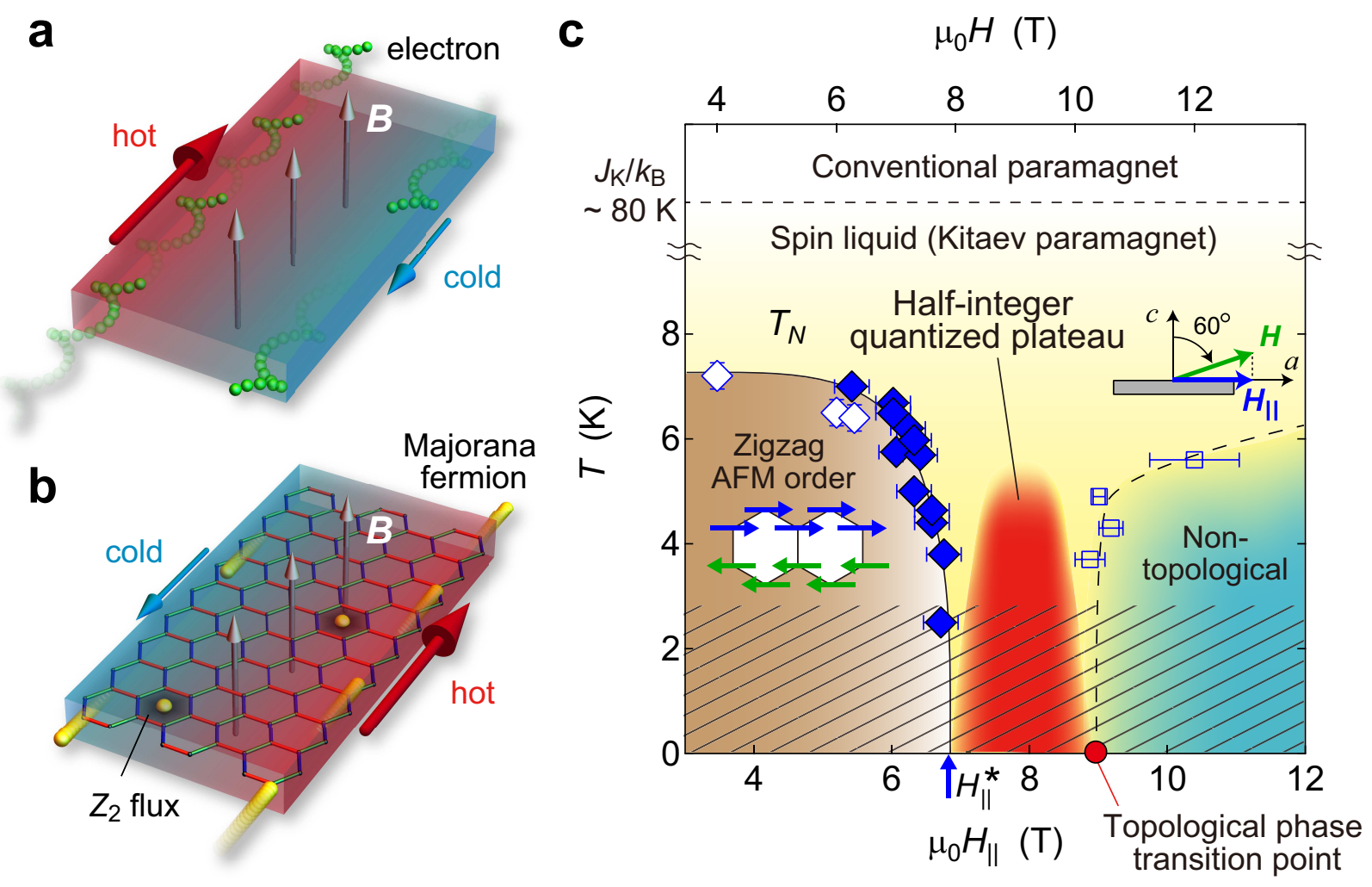

FIG. 1. 

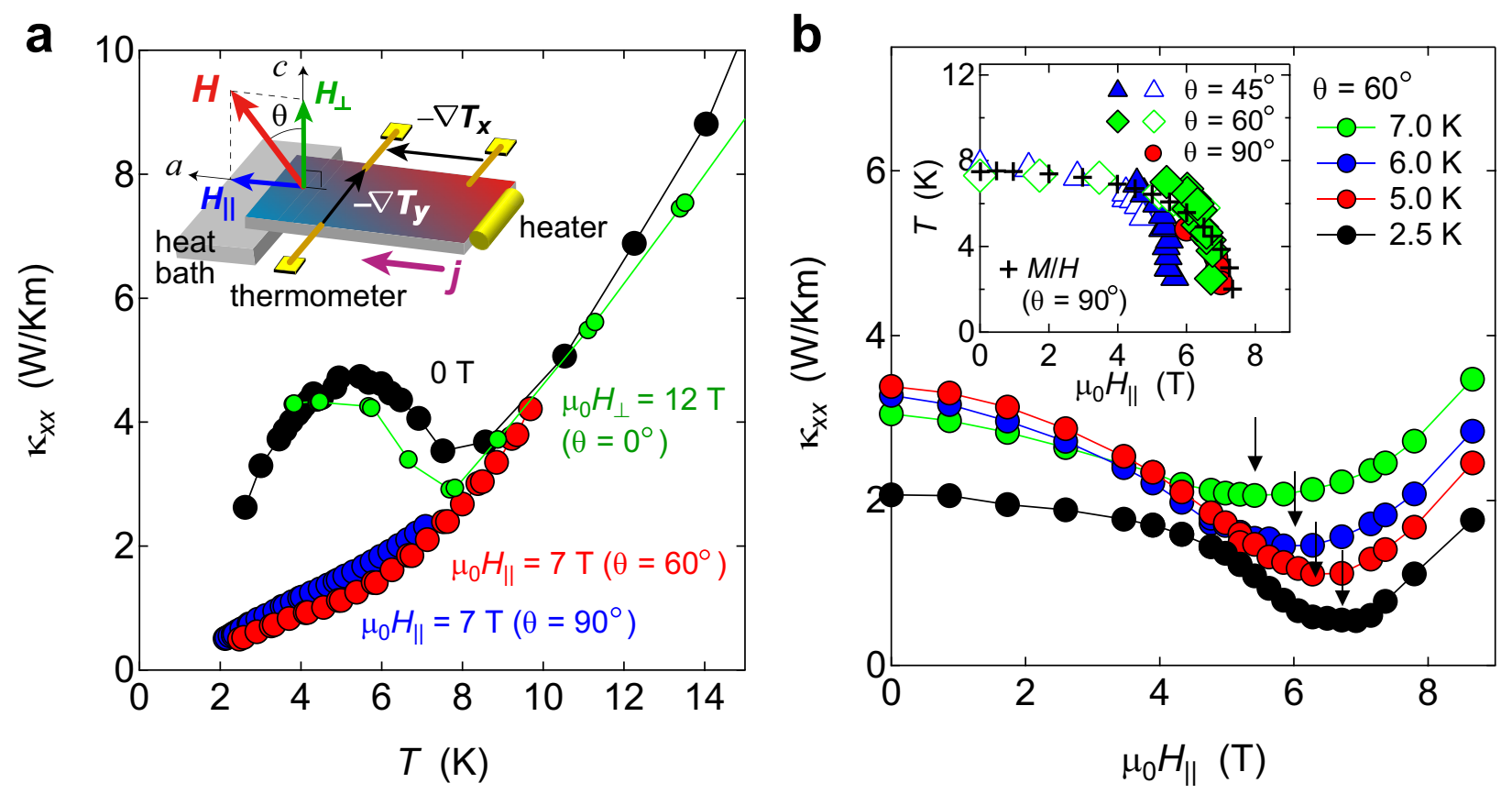

FIG. 2 . 


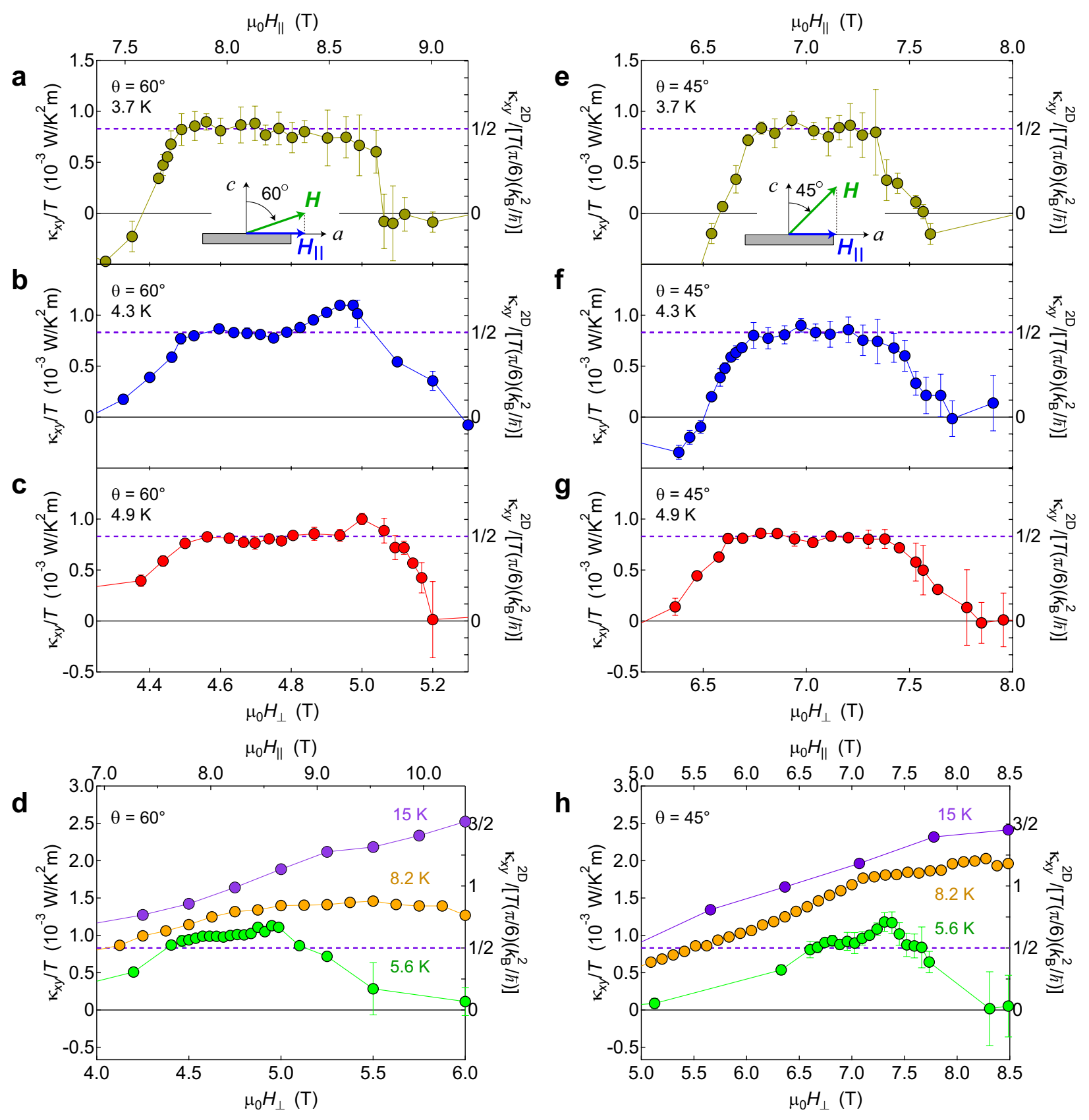

FIG. 3. 


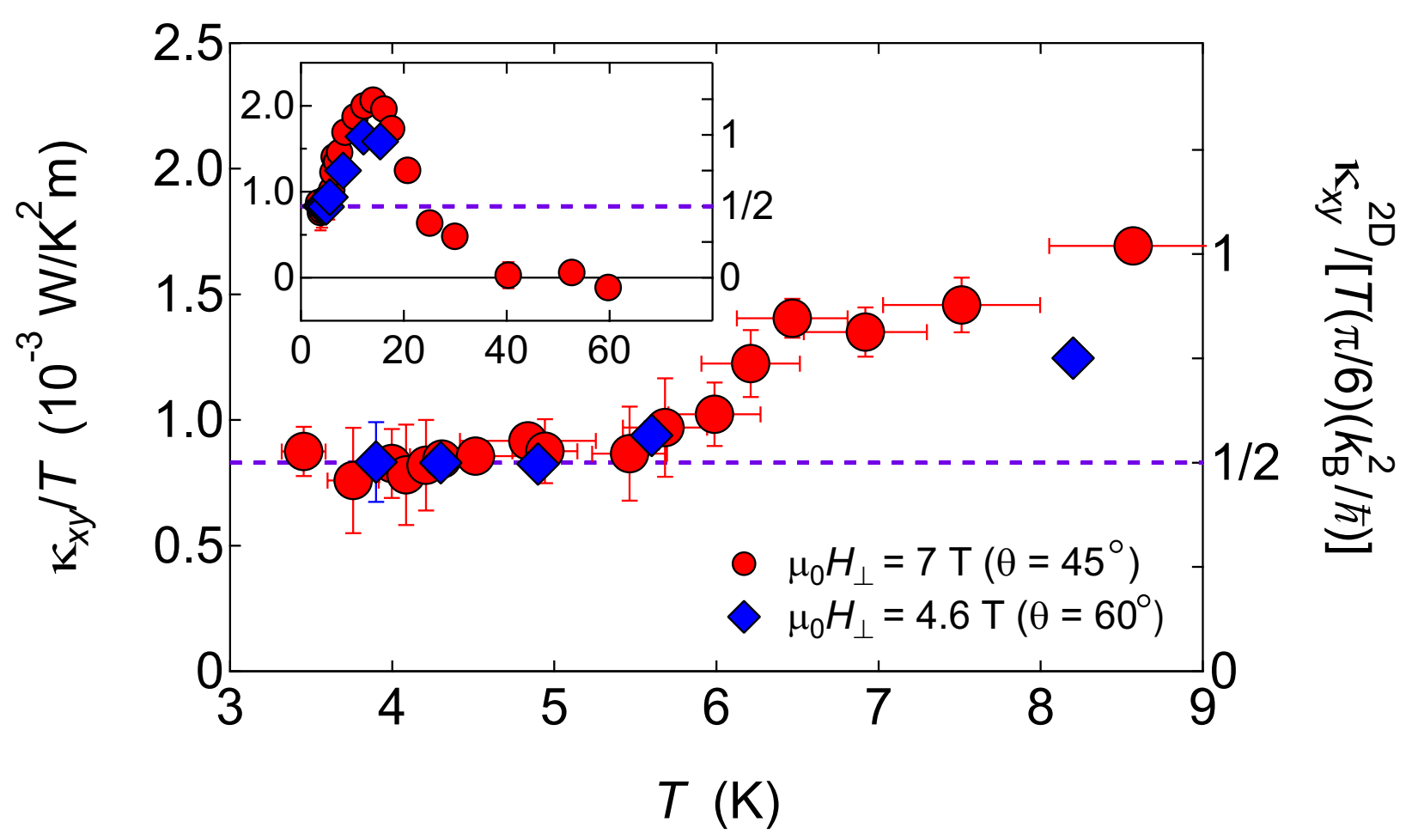

FIG. 4. 

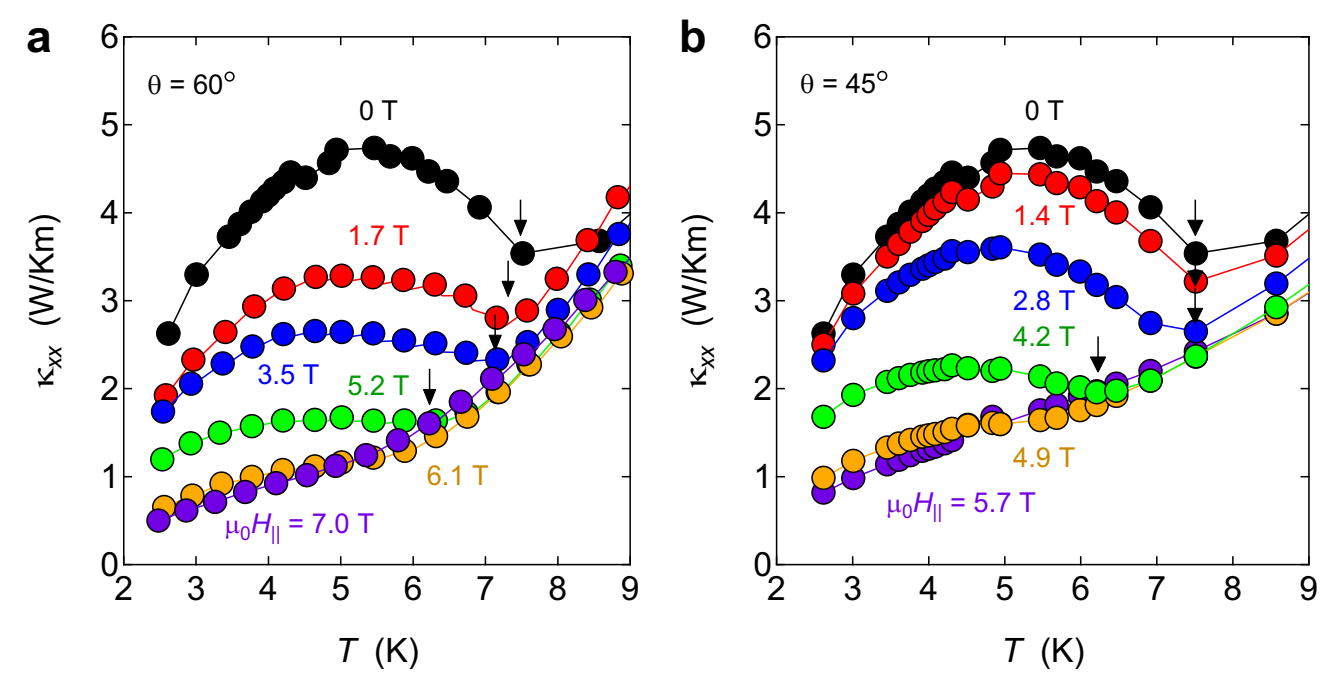

Extended Data Figure 1. 

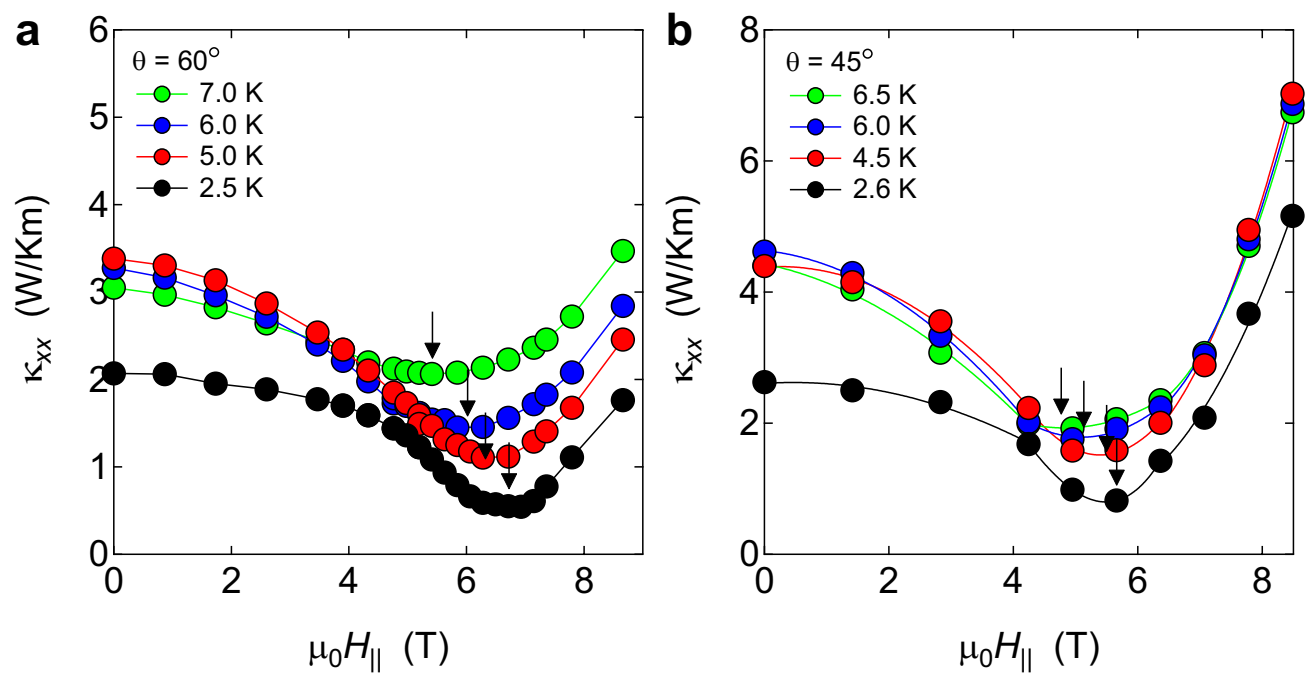

Extended Data Figure 2. 

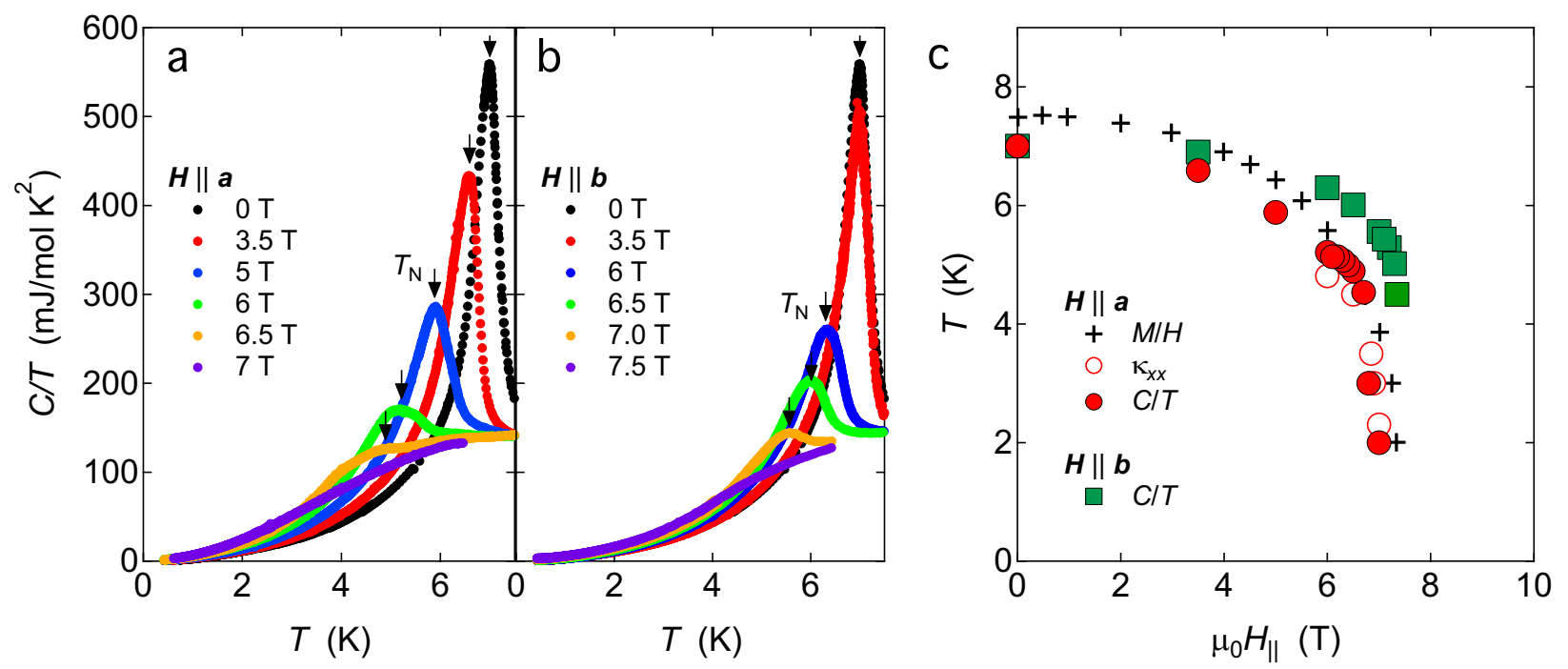

Extended Data Figure 3. 

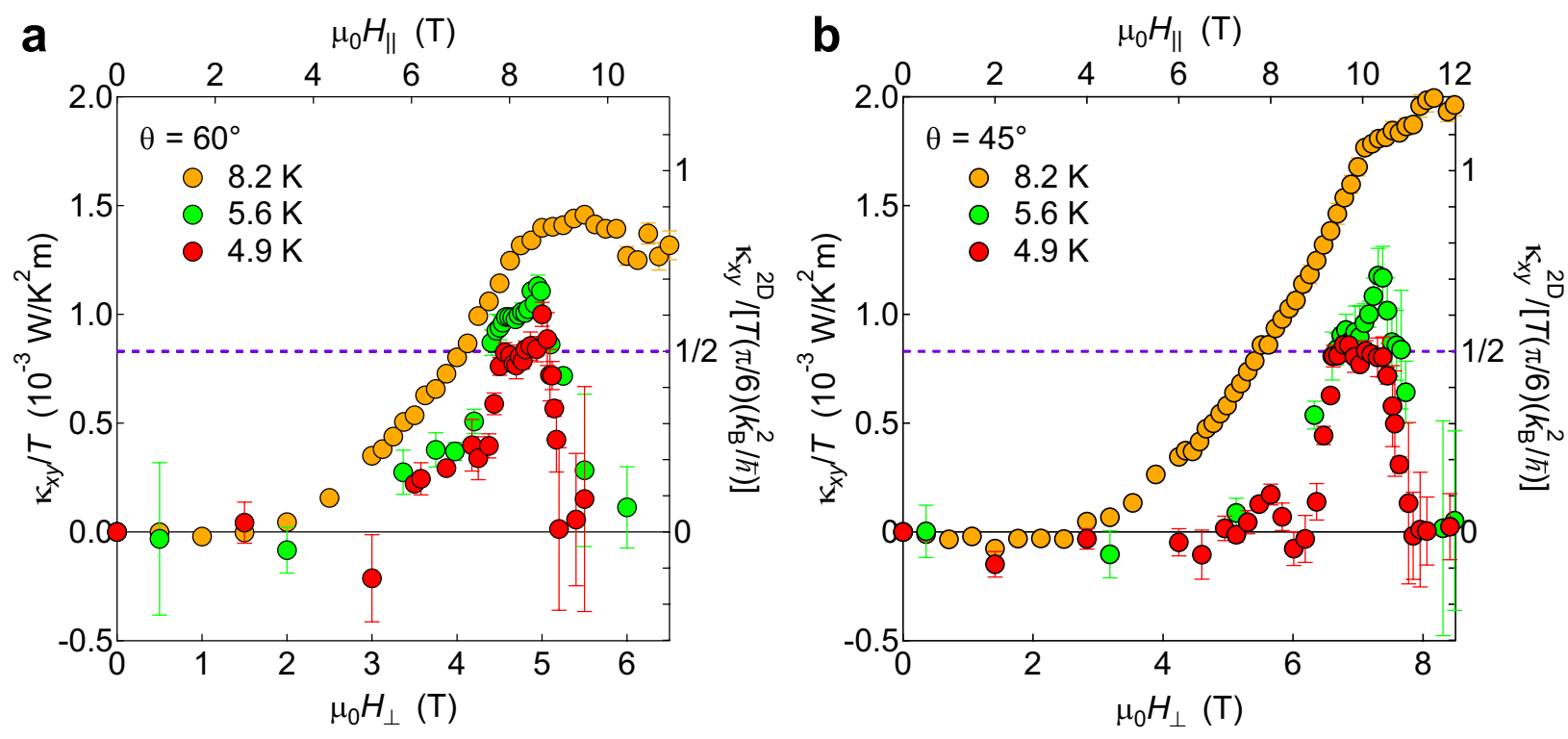

Extended Data Figure 4. 

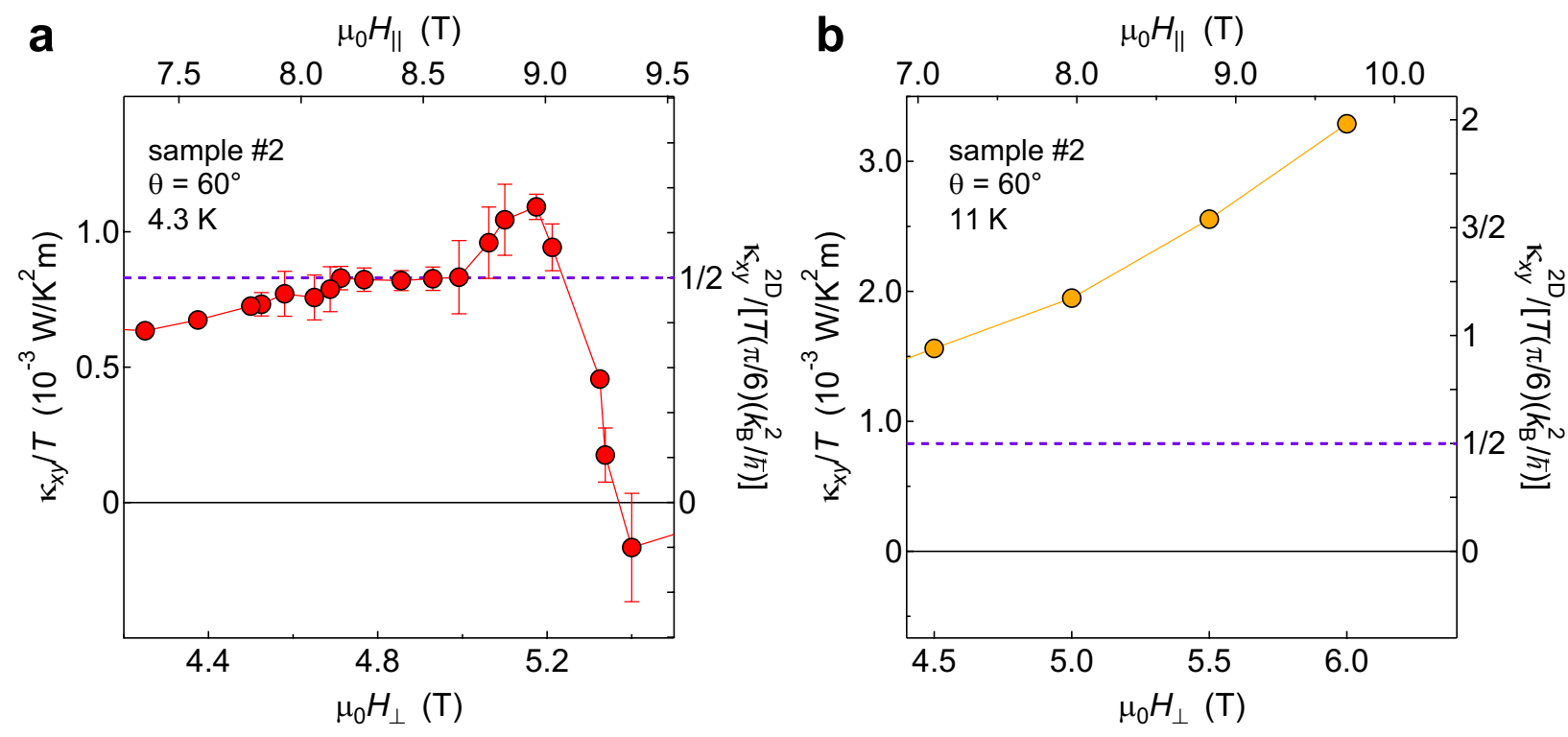

Extended Data Figure 5. 

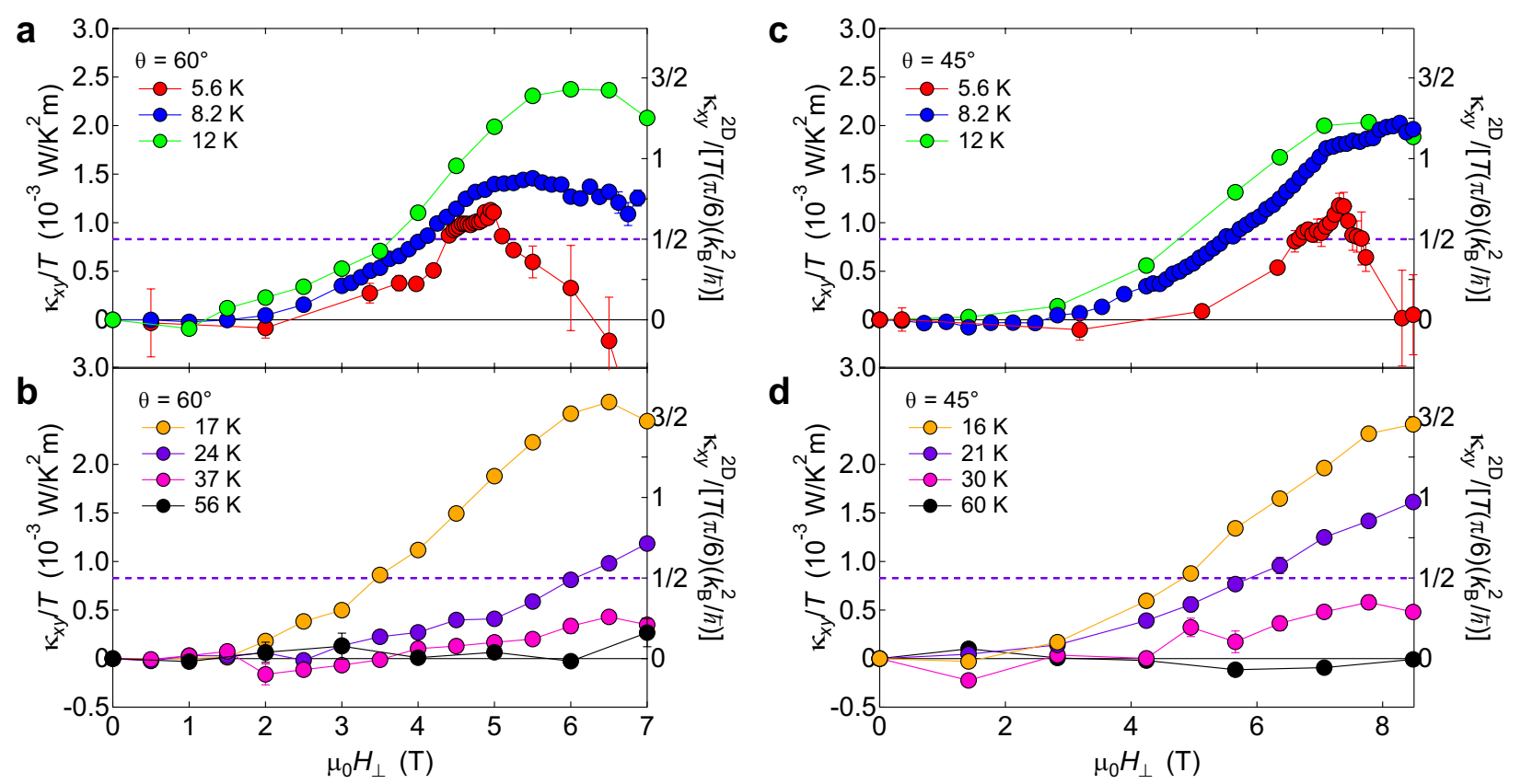

Extended Data Figure 6. 

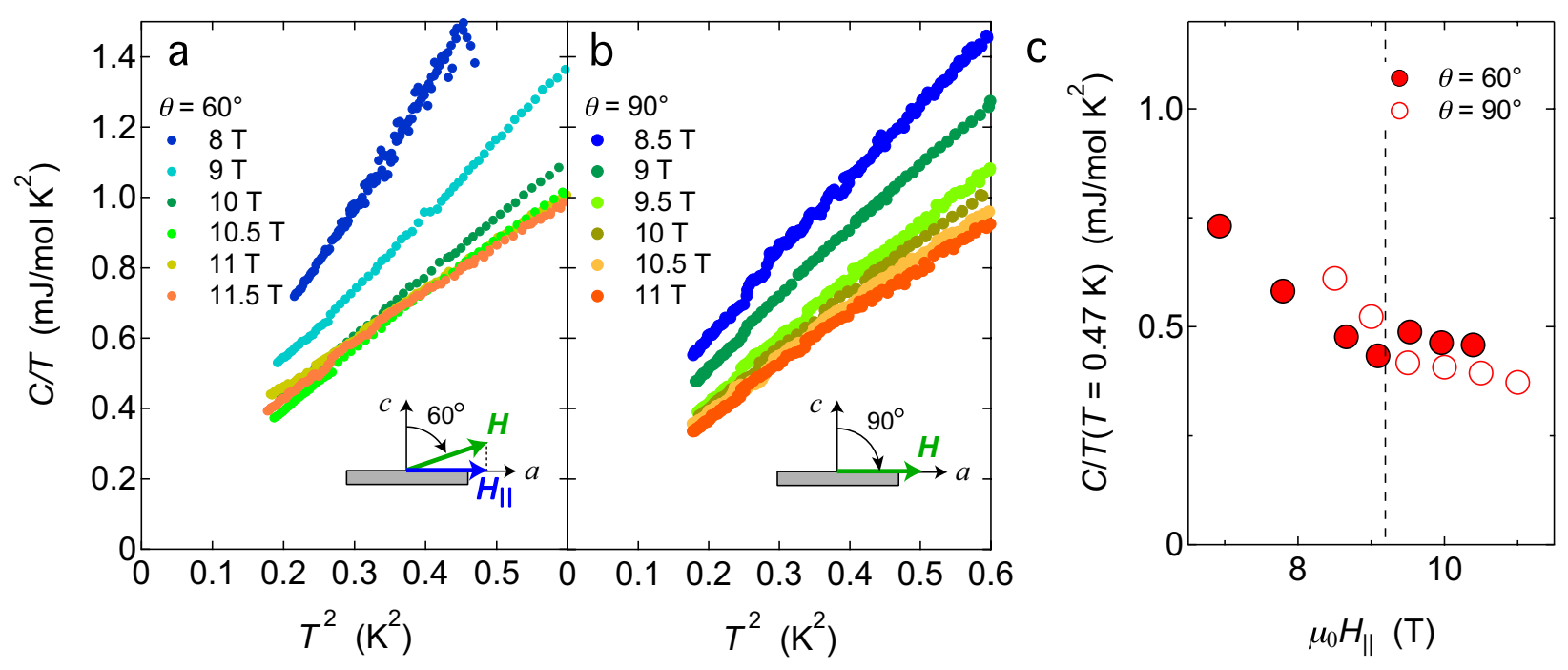

Extended Data Figure 7. 